from a direct straight reading. Two short appendices which explain the much-used concepts of the Dirac $\delta$ function and the Fourier transform are very worthwhile. Each chapter is well referenced up to 1971, and occasionally into 1972, and there are author and subject indices.

The book appears at an opportune moment when the Science Research Council has taken a third share in the Franco-German high-flux reactor at Grenoble and will have served a valuable purpose if it encourages British chemists to make use of the instruments which are now available to them there.

G. E. BACON

\section{Electrons and Holes}

Point Defects in Solids. Volume I. General and Ionic Crystals. Edited by James $\mathrm{H}$. Crawford, jun., and Lawrence M. Slifkin. Pp. $\mathrm{xv}+556$. (Plenum Press: New York and London, 1972.) $\$ 43$.

THERE are several stages in the excitation of electrons in ionic crystals, ranging from the closely bound electron-hole pair (exciton) to the complete separation of electrons and holes. In the pure crystal the electron, if it is free for sufficient time, may contribute to conduction. The transport occurs, however, not by a relatively free and simple particle, but by a "quasi-particle" comprising the electron and its associated cloud of polarization. The properties of polarons --electrons plus a pattern of ionic displacements-require a detailed knowledge of the interaction between electronic and vibrational excitations.

At sufficiently low temperatures the hole may immobilize itself by the polarization which it creates, producing a characteristic ESR spectrum. The whole phenomenon of the self-trapped hole is the more readily observed if there are deliberately added electron traps to give a sufficient hole lifetime.

Localization of electrons and holes also occurs at a wide variety of crystal imperfections-point defects, impurities and complexes involving either or both categories. The spectroscopy of these centres has reached a high level of sophistication. It covers electronic, spin and vibrational excitation of the centre and their interaction with the rest of the crystal. The techniques include those of unidirectional externally applied perturbations, such as electric, magnetic and stress fields, to determine the symmetry of the wave functions-a crucially important aspect of relating the structure of the defect to its atomic environment. In addition oscillatory perturbations have allowed complex modulation spectroscopy to be used both for microwave and optical spectra.

The electron centres are probably best known. They include the $F$ centre (electrons localized at halogen vacancy) and the $M$ centre (electrons localized at an associated pair of halogen vacancies). The hole centres were first thought to be complementary but have turned out to be quite different. They occur with extra halogens and uniaxial relaxations along the rows of halogen ions; the entity is a many-body collation of relaxed ions.

It is clear from the foregoing that the control of defects must be important. It is achieved by heat treatment to produce departures from stoichiometry, by the addition of differently charged substituted (aliovalent) ions (for example $\mathrm{Sr}^{2+}$ for $\mathrm{Na}^{+}$produces a further $\mathrm{Na}+$ vacancy) and by controlled irradiation. The defects and complexes have symmetries because of where they are situated in the lattice and these may be studied by relaxation methods-in an electric field (dielectric relaxation) or in a stress field (anelastic relaxation). These relaxation processes of complexes provide the microscopic mechanisms for larger scale mass transport (diffusion) studies.

Electrons trapped at such defects may give rise to very sharp absorption and emission spectral lines, as well as the associated (commoner) bands. The zeropoint uncertainties in the electronic states give a probability of a transition unbroadened by lattice vibrations (the zero-phonon spike, physically similar to Mössbauer $\gamma$-ray spike and band).

Underlying the whole subject is the knowledge of defect motion from the study of ionic conductivity at high temperatures, which is really where it all began. The ions needed space in which to move and intrinsic high temperature defects were adduced by Frenkel and Schottky to provide that space. The mass diffusion already referred to is also closely related to the ionic conduction process.

The subject is, therefore, a fascinating interplay of widely differing experimental and theoretical techniques. The present volume-the first of three setting out to provide a comprehensive discussion of the state of knowledge about point defects in solids-deals primarily with the subject outlined above, with a chapter on the statistical thermodynamics of defect formation and motion which provides a background for the whole series.

From the early days of the Göttingen work, advances have occurred, time and time again, as a result of carefully conceived experiments carried out with great sophistication on well characterized and controlled crystals. This comes through very clearly in the different sections. The sympathetic support of a number of theoretical physicists has led to the standard modern theory having been part of the jargon of the subject for a long time. An in- creasing physics interest in the field, apart from the desire comprehensively to understand a few simple crystals, is that it provides well defined experimental situations where theorists can test many-body approximations in systems with chosen degrees of coupling.

There is a remarkable continuing interest in this classic field of modern solid state physics and the present-expensive-volume will be a necessary addition to any library concerned with physics. The articles are well and authoritatively written and I would express only minor disappointment at the omission from so fine a volume of a discussion of the optical pumping via excited F-centre states and of the tunnelling phenomena between $F$ centres. E. W. J. Mitchei.I,

\section{Medicinal Plants}

Pharmacographia Indica. By William Dymock, C. J. H. Warden and David Hooper. Pp. 546. (The Institute of Health and Tibbi Research under the auspices of Hamdard National Foundation: Pakistan, 1972.) Pak. Rs. 220 ; US\$20.

THIs book has now been reprinted largely under the aegis of UNESCO following an international symposium on medicinal plants held in Peshawar in 1960. This is still the classical work of reference on the medicinal plants of the Indo-Pakistan subcontinent. Originally in three volumes, the Pharmacographia has now been produced in one volume by offset printing and the production is very good. Dymock and his colleagues, Warden and Hooper, produced these volumes in 1890 largely based on the smaller work relating to the medicinal plants of Western India and the drugs sold in that great drug market of the East, Bombay, produced in 1883 by Dymock. Dymock and his co-workers produced a great work of scholarship on the drugs and medicinal plants of India which up until then had been scattered through books and periodicals in many languages, but in addition where they appeared deficient in information they performed original investigations particularly towards elucidating the chemical composition and the physiological action of the plants and drugs. In this highly scientific work, however, Dymock felt that certain plants long used in India and of historical and mythological interest also had to be listed although they had little or no medicinal activity. The book is therefore a fascinating hotchpotch of superstition and science and not a little poetry. It is, of course, the intention to bring the work up to date but the editors and publishers are to be congratulated on bringing a rare work into more general circulation.

EDWARD Hi'TCHCOCK 